\title{
Premature Rupture of Membrane and Neonatal Infection
}

\author{
Laila Yahya A. Alhubaishi \\ Senior Specialist Register in Obstetrics \& Gynecology, Latifa Hospital, Dubai Health Authority, Duabi, United Arab Emirates \\ Email: atiffazari@hotmail.co.uk
}

How to cite this paper: Alhubaishi, L.Y.A. (2019) Premature Rupture of Membrane and Neonatal Infection. Open Journal of Obstetrics and Gynecology, 9, 1388-1391. https://doi.org/10.4236/ojog.2019.910134

Received: September 17, 2019

Accepted: October 22, 2019

Published: October 25, 2019

Copyright $\odot 2019$ by author(s) and Scientific Research Publishing Inc. This work is licensed under the Creative Commons Attribution International License (CC BY 4.0).

http://creativecommons.org/licenses/by/4.0/

\section{c) (i) Open Access}

\begin{abstract}
Premature rupture of membrane (PROM) is obstetric dilemma that carries risk of neonatal and maternal complications. PROM has controversy in management although many factors affect the outcome of PROM.

\section{Keywords}

Premature Rupture of Membrane, Perinatal Care, Neonatal Morbidity
\end{abstract}

\section{Background}

Premature rupture of membrane (PROM) is defined as rupture of the amniotic membranes before the onset of labor [1]. It occurs in approximately $10 \%$ of all pregnancies. When this event occurs before 37 weeks of gestation, it is deemed preterm premature rupture of membranes (PPROM) that has been estimated to affect $3 \%$ to $4.5 \%$ of all deliveries [1]. It was proven that high maternal and perinatal morbidity and mortality are associated with PROM [2]. Thus, it continues to be obstetric enigma in terms of causes and management despite advanced obstetric and perinatal care. PPROM leads to two main perinatal problems: prematurity and infection. Prematurity occurs because usually labor begins shortly after the rupture. Infection is the consequence of an open amniotic fluid cavity allowing germs to contaminate both the mother and the fetus.

Racial differences have been appreciated among women with PPROM. An increased incidence has been demonstrated specifically among black patients from $5.1 \%$ to $12.5 \%$ which is contrasted with corresponding white groups of $1.5 \%$ to $2.2 \%$ [3]. Socioeconomic parameters have not been found to directly influence the occurrence of PPROM. The role of smoking and sexual activity in producing PPROM is still points of some controversy. Deficiencies in vitamin C, copper, zinc, and overall nutritional status reflected by body mass index (BMI) have 
been associated with increased rates of PPROM. There seems to be a relatively strong association between vaginal bleeding and PPROM, with risk ranging between two folds and seven folds higher than control patients. Cervical parameters, multifetal pregnancy, poor obstetric history, preexisting medical conditions like maternal hypertension or diabetes and genital tract infection have been suggested to have some roles on PPROM [3].

The first problem relating to PROM seems to be infection, maternal and/or neonatal. The studies of JOHNSON and BLACKMON show that the later the PROM occurs during the pregnancy, the smaller is the overall risk of infection [4] [5].

Amniotic membranes insulate the fetus and amniotic fluid from microbial infections. The pathophysiology behind spontaneous PROM is idiopathic as far but it has been speculated that infection or degeneration may weaken the membranes and spontaneous rupture may occur. Amniotic membrane responds to various stimuli like membrane stretching or genital tract infections by producing mediators like prostaglandins, cytokines and other enzymes. A prolonged PROM is defined as rupture of membranes that persists for more than 24 hours before onset of labor. The interval between rupture of membrane and onset of labor is called latent period of leaking, which is considered as the cornerstone for the determination of maternal and neonatal outcomes [6].

PROM exposes the sterile intrauterine environment to pathogens in the vagina and environment. The most common organisms are Escherichia coli, Staphylococcus, Klebsiella and Pseudomonas [1]. Sharma S.K. et al. showed that out of all cases where vaginal swab culture grown organism, $83 \%$ of them were E. Coli and only 2 of them were sensitive to ampicillin [5].

Sequelae of amniotic membrane infection are potentially fatal for the pregnant women and their babies as well [7]. In 2005, the WHO reported that $37 \%$ of child mortality occurs below 5 years of age, and neonatal sepsis accounted for $29 \%$ of deaths within that age group [2]. An epidemiological study done by the WHO and UNICEF in 2010 found that there were 7.6 million cases of under five mortality, in which $64 \%$ occurred due to infection and the remaining $40.3 \%$ occurred in neonates [8]. Hurwitz A. et al. (2010) showed that clinical suspicion of chorioamnionitis is related with a higher incidence of neonatal infection but not with higher neonatal death rate [5].

Other complications for the newborn consist of fetal distress, cord compression, deformation, altered pulmonary development, respiratory distress syndrome, septicemia, meningitis, pneumonia etc. The knowledge of incidence of early onset neonatal sepsis in relation to PROM and its effect on neonatal outcome is essential in order to prevent the neonatal morbidity and mortality. Diagnosis of early onset sepsis by close observation for early signs of sepsis, aggressive evaluation and early treatment has decreased the incidence of early onset sepsis associated with PROM [1]. The incidence of perinatal mortality was 5\% with the most common causes being neonatal sepsis and pneumonia. Moreover, the lesser the time interval between the rupture of amniotic membrane and de- 
livery, the lesser was the maternal and perinatal morbidity and mortality [9].

The controversy about the benefit versus the risk of delaying labor after PROM is the main problem concerning the management of PROM. According to the literature, the risk of chorioamnionitis is variously related to the latency period: many authors find no relationship [4] [10] [11] while others find a negative relationship [12]. Similar controversial results are found about the incidence of neonatal infection, but the actual conclusion seems to be that there is no relationship between the length of the latency period and the neonatal infection.

A multicenter study on PROM in term pregnancy, conducted in the US, Canada, UK, and Israel, found that prolonged rupture of membrane for 48 hours and $24-48$ hours increases the risk of neonatal infection by 2.25 times [7]. In addition, babies with neonatal sepsis were hospitalized for 32 days on average, compared to 3 days in babies without the condition. For this reason, PROM adds more to the financial burden on the health care system.

\section{Conclusion}

Premature rupture of membrane (PROM) is one of medical challenges with neonatal short and long life morbidities with mortality. It has different aetiologias. Improvement of neonatal care facilities plays a crucial role in better outcome.

\section{Conflicts of Interest}

The author declares no conflicts of interest regarding the publication of this paper.

\section{References}

[1] Ramesh, T.V., Panigrahi, B., Pranaya, P. and Bindu, P.H. (2018) Outcome of Neonates Born to Mothers with Premature Rupture of Membranes. International Journal of Contemporay Pediatrics, 5, 1190-1194. https://doi.org/10.18203/2349-3291.ijcp20181994

[2] Surayapalem, S., Cooly, V. and Salicheemala, B. (2017) A Study on Maternal and Perinatal Outcome in Premature Rupture of Membranes at Term. International Journal of Reproduction, Contraception, Obstetrics and Gynecology, 6, 5368-5372. https://doi.org/10.18203/2320-1770.ijrcog20175244

[3] Killbride, H.W., Yeast, J.D. and Thibealt, D.W. (1989) Intrapartum and Delivery Room Management of Premature Ruture of Membranes Complicated by Olygohydramnios. Clinics in Perinatology, 16, 863-888. https://doi.org/10.1016/S0095-5108(18)30608-0

[4] Johnson, J.W. and Daikoku, N.H. (2012) Prepartal Rupture of Thechorioamnion. Birth Defects, 21.

[5] Blackmon, L., Alger, L. and Crenshaw, C. (2006) Fetal and Neonatal Outcomes Associated with Premature Rupture of the Membranes. Clinical Obstetrics and Gynecology, 29, 779-815. https://doi.org/10.1097/00003081-198612000-00007

[6] Sharma, S.K. and Dey, M. (2017) Maternal and Neonatal Outcome in Cases of Premature Rupture of Membranes beyond 34 Weeks of Gestation. International Jour- 
nal of Reproduction, Contraception, Obstetrics and Gynecology, 6, 1302-1305. https://doi.org/10.18203/2320-1770.ijrcog20171382

[7] Oviyanti, D. and Wahono, W.T. (2018) Risk Factors for Neonatal Sepsis in Pregnant Women with Premature Rupture of the Membrane. Journal of Pregnancy, 2018, Article ID: 4823404. https://doi.org/10.1155/2018/4823404

[8] Liu, L., Johnson, H.L., Cousens, S., et al. (2012) Global, Regional and National Causes of Child Mortality: An Update Systematic Analysis for 2010 with Time Trends Since 2000. The Lancet, 379, 2151-2161. https://doi.org/10.1016/S0140-6736(12)60560-1

[9] BPS SI-. BPS-, BKKBN/Indonesia NP and FPB-, Health/Indonesia KK-. K-. Mof, International ICF. Indonesia Demographic and Health Survey 2012. Jakarta, Indonesia: BPS, BKKBN, Kemenkes, and ICF International. 2013.

[10] Hnat, M.D., Mercer, B.M., Thurnauet, G., et al. (2005) Perinatal Outcomes in Women with Preterm Rupture of Membranes between 24 and 32 Weeks of Gestation and a History of Vaginal Bleeding. American Journal of Obstetrics \& Gynecology, 193, 164-168. https://doi.org/10.1016/j.ajog.2004.10.625

[11] Broekhuizen, F.F., Gilman, M. and Hamilton, P.R. (1985) Amniocentesis for Gram Stain and Culture in Preterm Premature Rupture of the Membranes. Obstetrics Gynecology, 66, 316-321.

[12] Gonik, B., Bottoms, S.F. and Cotton, D.B. (1985) Amniotic Fluid Volume as a Risk Factor in Preterm Premature Rupture of the Membranes. Obstetrics Gynecology, 65, 456-459. 